\title{
Final analysis of the randomised PEAK trial: overall survival and tumour responses during first-line treatment with mFOLFOX6 plus either panitumumab or bevacizumab in patients with metastatic colorectal carcinoma
}

\author{
Fernando Rivera ${ }^{1}$ - Meinolf Karthaus ${ }^{2} \cdot$ J. Randolph Hecht ${ }^{3}$ - Isabel Sevilla ${ }^{4}$. \\ Frédéric Forget $^{5}$ - Gianpiero Fasola ${ }^{6}$. Jean-Luc Canon ${ }^{7} \cdot$ Xuesong Guan $^{8}$. \\ Gaston Demonty ${ }^{9} \cdot$ Lee S. Schwartzberg ${ }^{10}$
}

Accepted: 22 March 2017 / Published online: 19 April 2017

(C) The Author(s) 2017. This article is an open access publication

\begin{abstract}
Purpose To report planned final overall (OS) and progression-free survival (PFS) analyses from the phase II PEAK trial (NCT00819780).

Methods Patients with previously untreated, KRAS exon 2 wild-type (WT) metastatic colorectal cancer (mCRC) were randomised to mFOLFOX6 plus panitumumab or bevacizumab. The primary endpoint was PFS; secondary endpoints included OS, objective response rate, duration of response (DoR), time to response, resection and safety.
\end{abstract}

Electronic supplementary material The online version of this article (doi:10.1007/s00384-017-2800-1) contains supplementary material, which is available to authorized users.

Fernando Rivera

oncrhf@humv.es

1 Hospital Universitario Marqués de Valdecilla, Av. de Valdecilla, 39008 Santander, Spain

2 Städtisches Klinikum München, Klinikum Neuperlach, Munich, Germany

3 David Geffen School of Medicine, University of California Los Angeles, Los Angeles, CA, USA

4 Virgen de la Victoria University Hospital, Malaga, Spain

5 Centre Hospitalier de l'Ardenne, Libramont, Belgium

6 University Hospital Santa Maria della Misericordia, Udine, Italy

7 Grand Hôpital de Charleroi, Charleroi, Belgium

8 Amgen Inc., Biostatistics, Thousand Oaks, CA, USA

9 Medical Development - Oncology, Amgen (Europe) GmbH, Zug, Switzerland

10 West Clinic, Memphis, TN, USA
Treatment effect by tumour $R A S$ status was a prespecified objective. Exploratory analyses included early tumour shrinkage (ETS) and depth of response (DpR).

Results One hundred seventy patients had RAS WT and 156 had $R A S$ WT/BRAF WT mCRC. Median PFS was longer for panitumumab versus bevacizumab in the $R A S$ WT ( 12.8 vs 10.1 months; hazard ratio (HR) $=0.68[95 \%$ confidence intervals $(\mathrm{CI})=0.48-0.96] ; p=0.029)$ and $R A S \mathrm{WT} / B R A F \mathrm{WT}$ (13.1 vs 10.1 months; $\mathrm{HR}=0.61[95 \% \mathrm{CI}=0.42-0.88]$; $p=0.0075)$ populations. Median OS (68\% OS events) for panitumumab versus bevacizumab was 36.9 versus 28.9 months $(\mathrm{HR}=0.76[95 \% \mathrm{CI}=0.53-1.11] ; p=0.15)$ and 41.3 versus 28.9 months ( $\mathrm{HR}=0.70$ [95\% CI $=0.48$ 1.04]; $p=0.08$ ), in the RAS WT and RAS WT/BRAF WT populations, respectively. Median DoR (11.4 vs 9.0 months; $\mathrm{HR}=0.59[95 \% \mathrm{CI}=0.39-0.88] ; p=0.011)$ and $\mathrm{DpR}(65.0$ vs $46.3 \%$; $p=0.0018$ ) were improved in the panitumumab group. More panitumumab patients experienced $\geq 30 \%$ ETS at week 8 (64 vs $45 \%$; $p=0.052$ ); ETS was associated with improved PFS/OS. No new safety signals occurred.

Conclusions First-line panitumumab + mFOLFOX6 increases PFS versus bevacizumab + mFOLFOX6 in patients with RAS WT mCRC.

Keywords Bevacizumab $\cdot$ First-line $\cdot$ Metastatic colorectal cancer $\cdot$ Overall survival $\cdot$ Panitumumab

\section{Introduction}

First-line treatments for patients with metastatic colorectal cancer (mCRC) comprise chemotherapy combined with an epidermal growth factor receptor inhibitor (EGFRI), 
panitumumab or cetuximab, or the vascular endothelial growth factor inhibitor (VEGFI) bevacizumab [1]. Confirmation of wild-type (WT) tumour $R A S$ status is essential ahead of prescribing EGFRIs [1,2], the importance of evaluating tumour $R A S$ status ahead of bevacizumab treatment is less clear [3, 4]. In the EU, panitumumab is indicated in combination with FOLFOX or FOLFIRI for the first-line treatment of patients with RAS WT (no mutations in exons 2, 3 and 4 of $K R A S$ and NRAS) mCRC [5]. In the USA, panitumumab is indicated in KRAS (exon 2) WT mCRC combined with FOLFOX for first-line treatment, and as monotherapy following disease progression after prior treatment with fluoropyrimidine, oxaliplatin and irinotecancontaining chemotherapy [6]. Bevacizumab is also indicated in the EU for the treatment of patients with mCRC in combination with fluoropyrimidine-based chemotherapy [7] and in the USA with intravenous 5-fluorouracil-based chemotherapy for first- or second-line mCRC treatment and with fluoropyrimidine-irinotecan- or fluoropyrimidine-oxaliplatinbased chemotherapy for second-line treatment after progression (PD) on a first-line bevacizumab-containing regimen [8].

PEAK (Panitumumab Efficacy in combination with mFOLFOX6 Against bevacizumab plus mFOLFOX6 in mCRC subjects with KRAS WT tumours) is a phase II, randomised study evaluating the efficacy and safety of firstline panitumumab + modified FOLFOX6 (mFOLFOX6) versus bevacizumab + mFOLFOX6 in patients with KRAS exon 2 WT mCRC [9]. The primary objective was to assess progression-free survival (PFS) in the KRAS exon 2 WT population; a prespecified secondary objective was to assess PFS and overall survival (OS) in patients with RAS WT mCRC. In the primary analysis of PEAK, first-line panitumumab + mFOLFOX6 was associated with numerically longer OS than bevacizumab + mFOLFOX6 in patients with RAS WT mCRC ( $41 \%$ OS events; median OS = 41.3 vs 28.9 months; hazard ratio $(\mathrm{HR})=0.63[95 \%$ confidence intervals $(\mathrm{CI})=0.39$ 1.02]; $p=0.058$ ) and significantly longer PFS (median $\mathrm{PFS}=13.0$ vs 9.5 months; $\mathrm{HR}=0.65[95 \% \mathrm{CI}=0.44$ 0.96 ]; $p=0.029$ ) [9]. Overall response rates (ORR) by Response Evaluation Criteria In Solid Tumours (RECIST) were similar between treatments (63.6 vs $60.5 \%$, for panitumumab vs bevacizumab groups, respectively) [9]. RECIST, however, does not fully take into account timing, depth (DpR) and duration of response (DoR), all of which may influence long-term outcomes [10,11].

In addition to $R A S$ mutations, other biomarkers have potential predictive and prognostic importance in mCRC. $B R A F$ mutation status has emerged as a strong prognostic marker [12] but there is a lack of evidence to support best approaches to treatment for BRAF mutant (MT) mCRC [1, 2]. Clinical trials may, therefore, be the best option for some patients. Alternatively, a small population sub-analysis from the TRIBE trial suggested a potential OS benefit for patients with $B R A F$ mutations receiving bevacizumab + FOLFOXIRI $(n=16)$ versus bevacizumab + FOLFIRI $(n=12)$ [13], and so, this combination may be an option for patients with good performance status.

Here, we report updated OS and PFS from a planned final analysis of PEAK data (RAS WT and RAS WT/BRAF WT populations). Exploratory analyses of tumour assessments beyond RECIST were also performed, focussing on tumour dynamics (early tumour shrinkage (ETS), depth of response (DpR) and changes in tumour load over time (RAS WT population only)).

\section{Material and methods}

\section{Study design and patients}

PEAK (NCT00819780) was a phase II, randomised (1:1), open-label first-line study in patients with previously untreated KRAS exon 2 WT mCRC. Treatment continued until $\mathrm{PD}$, unacceptable toxicity, death, consent withdrawal, or investigator decision. The trial was conducted in compliance with the Declaration of Helsinki. The study protocol was approved by an independent ethics committee at each participating study centre. All patients provided informed consent before any study procedures were performed.

Key eligibility criteria for PEAK (primary analysis) included age $\geq 18$ years; an Eastern Cooperative Oncology Group performance score of $0 / 1$; histologically/cytologically confirmed $\mathrm{mCRC}$ with unresectable metastatic disease; KRAS exon 2 (codons 12 and 13) WT tumour status; one unidimensionally measurable lesion of $\geq 20 \mathrm{~mm}$ (per modified RECIST guidelines); and no prior chemotherapy, anti-EGFR therapy or bevacizumab therapy for $\mathrm{mCRC}$.

\section{Extended $R A S$ analyses and $B R A F$ mutation analyses}

An extended $R A S$ analysis was prespecified in PEAK [9]. In brief, banked tumour samples for patients with KRAS exon 2 WT tumours were tested for prespecified mutations in NRAS exon 2 (codons 12 and 13), and KRAS and NRAS exons 3 (codons 59 and 61) and 4 (codons 117 and 146). Mutations were also assessed in exon 15 (codon 600) of BRAF (exploratory analysis). Mutation status was assessed using bidirectional Sanger sequencing and WAVE-based SURVEYOR ${ }^{\circledR}$ scan kits (Transgenomic; Omaha, NE, USA), as reported previously [12].

\section{Efficacy assessments}

Endpoints reported here include PFS, OS, ORR, DoR, time to response (TTR), resection rates, DpR, change in tumour load versus baseline (over time), and ETS. Tumour response was 
assessed by the investigator every 8 weeks ( \pm 7 days) using modified RECIST (version 1.0; there was no independent review). ETS was defined as the proportion of patients with tumour shrinkage at week 8 ; two cutoff values were used, $\geq 30$ and $\geq 20 \%$. Tumour shrinkage was defined as the change in sum of the longest diameters (SLD) of all target lesions. DpR was defined for each patient as the percentage of tumour shrinkage at nadir or progression. DpR has a positive value for tumour reduction, a negative value for tumour growth and is zero for no change. If shrinkage occurred during treatment, then DpR was the greatest percentage tumour shrinkage observed at nadir versus baseline. In all other cases, DpR was defined as the percentage of tumour change at progression. If progression did not occur, DpR was not defined as PFS could not be defined. Changes in tumour load were calculated as mean $(95 \% \mathrm{CI})$ percentage change from baseline in the SLD of all target lesions over time.

\section{Safety assessments}

Patients were followed-up for safety until 30 days after the last dose of study drug. Adverse events (AEs) were graded using Common Terminology Criteria for Adverse Events (version 3.0), with modifications for specific skin- and nail-related toxicities.

\section{Statistical analyses}

The primary endpoint in PEAK was PFS; secondary endpoints included OS, ORR, DoR, TTR, resection rates, and safety (all reported here for the RAS WT population). Sixmonth PFS and 2-year OS rates by resection status were calculated by treatment (exploratory analysis). Exploratory analyses of DpR, changes in tumour load versus baseline and ETS, were performed for the RAS WT population; median PFS and OS were calculated for patients with/without $\geq 30$ and $\geq 20 \%$ ETS, by treatment and overall. Top-line efficacy results (PFS, OS and ORR) were also reported for the RAS WT/BRAF WT and $R A S \mathrm{WT} / B R A F$ MT populations.

The treatment effect HR for panitumumab relative to bevacizumab combined with mFOLFOX6 and the associated 95\% CIs were estimated from a Cox model stratified by previous adjuvant oxaliplatin therapy. Kaplan-Meier curves were generated for all time-to-event endpoints.

The primary analysis of PEAK was performed in 2012 when the target of $\sim 168$ PFS events was achieved [9]. This PFS event goal was expected to be achieved with recruitment of approximately 280 patients. The current final analysis was planned to occur 36 months after the last patient enrolled. No formal hypothesis testing was planned; all reported $p$ values are descriptive.

The prespecified extended $R A S$ analysis included all intentto-treat (ITT) patients with $R A S$ data available. The extended
$R A S$ statistical analysis plan was developed before the data analysis was conducted and no biomarker testing results were available to the study team before plan approval.

\section{Results}

\section{Patients}

Between April 2009 and December 2011, 285 patients with $K R A S$ exon 2 WT mCRC were enrolled from 60 sites; 142 patients were randomly assigned to panitumumab + mFOLFOX6 and 143 to bevacizumab + mFOLFOX6 (primary analysis ITT set [Fig. 1]). Overall, 278 patients received treatment. Samples from 250 patients in the ITT population (88\%) underwent extended $R A S$ analysis, with 233 $(82 \%)$ having a result. Twelve patients were identified as having $K R A S$ exon 2 mutations upon additional testing, giving a $K R A S$ exon 2 WT population of 221. Among these 221 patients, 170 (77\%) had RAS WT mCRC. BRAF mutations were found in tumours from 14 patients (panitumumab + mFOLFOX6 $n=11$; bevacizumab $+\operatorname{mFOLFOX6~} n=3$ ), all of whom had RAS WT mCRC.

Baseline demographics and disease characteristics were generally similar between treatments in both the RAS WT and RAS WT/BRAF WT populations (Table 1). The mean (standard deviation) actual follow-up time was 134.1 (73.8) weeks in the panitumumab arm and 115.7 (71.0) weeks in the bevacizumab arm. Overall, the median duration of panitumumab treatment was 14.0 cycles in both the $R A S$ WT and RAS WT/BRAF WT populations. The median duration of bevacizumab treatment was 11.0 and 11.5 cycles in the $R A S \mathrm{WT}$ and $R A S \mathrm{WT} / B R A F \mathrm{WT}$ populations, respectively.

\section{Efficacy}

At the time of analysis, 73 versus $85 \%$ of patients with $R A S$ WT mCRC receiving panitumumab + mFOLFOX6 versus bevacizumab + mFOLFOX6, respectively, had PFS events (Table 2). In the RAS WT population, PFS was longer in the panitumumab versus bevacizumab group (median $=12.8 \mathrm{vs}$ 10.1 months; $\mathrm{HR}=0.68$ [95\% CI $=0.48-0.96] ; p=0.0292$ ) (Fig. 2a).

Mortality rates in the RAS WT population at the time of analysis were 65 versus $71 \%$ for the panitumumab + mFOLFOX6 versus bevacizumab + mFOLFOX6 groups, respectively (Table 2). In the RAS WT population, median OS was 36.9 versus 28.9 months in the panitumumab versus bevacizumab groups, respectively (HR $=0.76(95 \%$ $\mathrm{CI}=0.53-1.11)$ ); $p=0.15$ ) (Fig. 2b). Although some patient subpopulations were small, the trend (not statistically significant) towards improved OS in the panitumumab group was generally observed across subpopulations defined by baseline 
Fig. 1 PEAK study CONSORT diagram (data cutoff 11 February 2015)

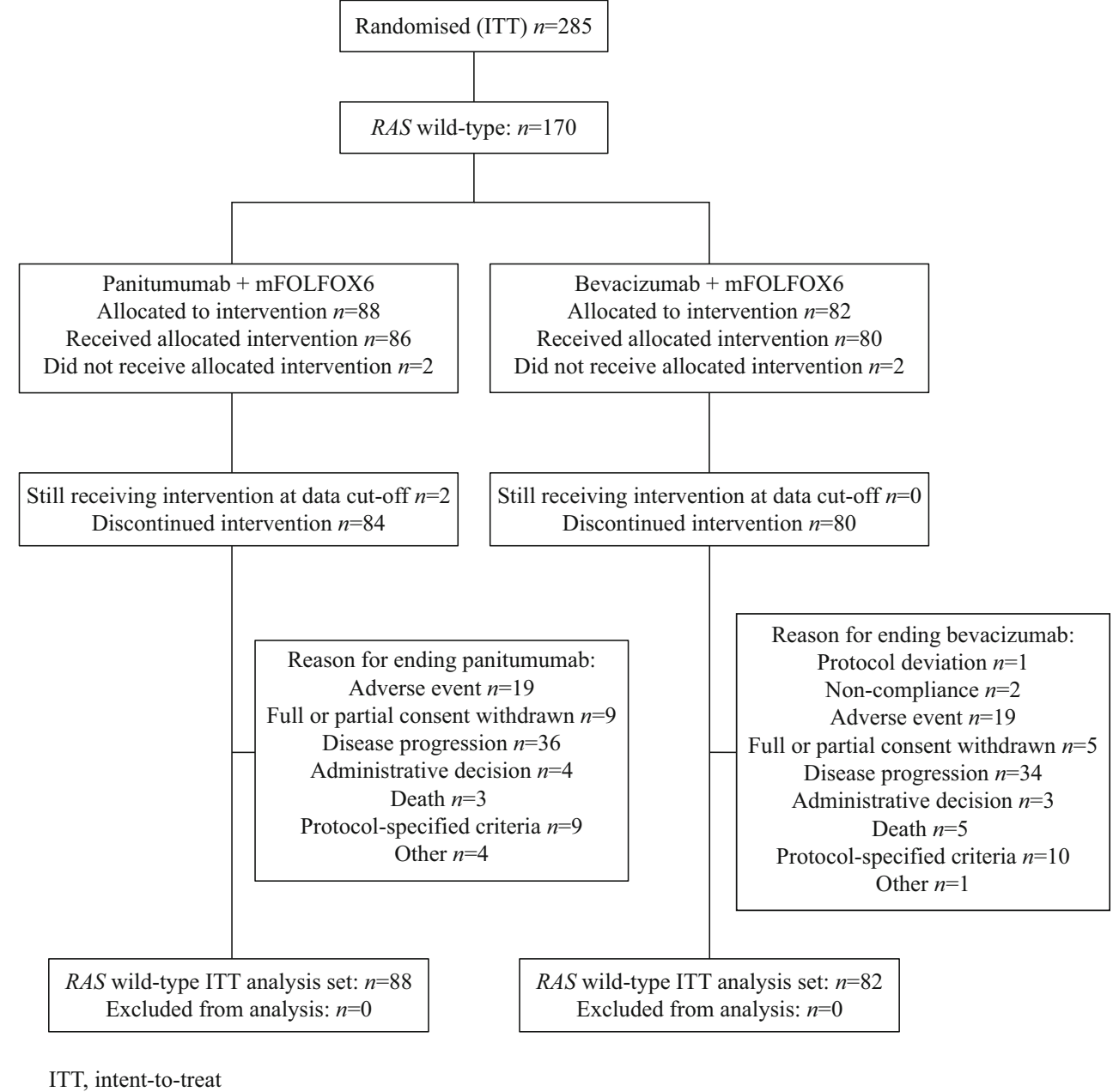

covariates (Online Resource Fig. A1). Similar proportions of patients in the panitumumab and bevacizumab groups subsequently crossed over to anti-VEGF and anti-EGFR therapies, respectively (Table 2 ).

Overall, 169 patients with $R A S$ WT mCRC were included in the ORR analysis; ORRs were similar in the panitumumab + mFOLFOX6 and bevacizumab + mFOLFOX6 arms (65 vs $60 \%$, respectively; odds ratio $=1.12(95 \% \mathrm{CI}=0.56-2.22)$; $p=0.86$ ) (Table 2).

Similar observations for PFS (median $=13.1 \mathrm{vs}$ 10.1 months; HR $=0.61(95 \% \mathrm{CI}=0.42-0.88)$; $p=0.0075$ ), OS (median $=41.3$ vs 28.9 months; $\mathrm{HR}=0.70$ (95\% CI $=0.48-1.04) ; p=0.08)$ and ORR (65 vs 62\%) were found for the panitumumab versus bevacizumab groups, respectively, in the $R A S \mathrm{WT} / B R A F$ WT population (Table 2; Online Resource Fig. A2). The efficacy of panitumumab and bevacizumab in patients with $R A S \mathrm{WT} / B R A F \mathrm{MT}$ disease could not be determined due to the small number of patients in this subpopulation ( $n=11$ and $n=3$, respectively; Online Resource Table A1).

In patients with $R A S$ WT mCRC, median TTR was numerically shorter, median DoR longer and median DpR greater in the panitumumab + mFOLFOX6 versus bevacizumab + mFOLFOX6 group (Table 3). Resection rates and outcomes following resection were similar between treatments for the $R A S$ WT population, although sample sizes were small for this analysis (Table 3 ).

The mean percentage change from baseline in tumour load in the RAS WT population appeared to favour panitumumab at all measured time points, although the CIs were wide (Fig. 3). Figure 3 shows tumour data at each scheduled visit; following PD, patients were only followed up for survival and no further CT scans were analysed.

Overall, 154 patients with RAS WT mCRC had tumour shrinkage data available at baseline and week 8. Compared with the bevacizumab + mFOLFOX6 arm, more patients in the panitumumab + mFOLFOX6 group experienced $\geq 30 \%$ ETS (Table 4). Numerically, more patients in this group also had $\geq 20 \%$ ETS. For those achieving $\geq 30$ or $\geq 20 \%$ ETS, numerically longer PFS and OS were seen in the panitumumab versus bevacizumab arm (Table 4; Online Resource Fig. A3). ETS ( $\geq 30$ or $\geq 20 \%$ ) was associated with numerically longer PFS and OS, irrespective of treatment received (Online Resource Table A2). When treatment arms 
Table 1 Baseline demographics and disease characteristics

\begin{tabular}{|c|c|c|c|c|}
\hline & \multicolumn{2}{|l|}{$R A S$ wild-type } & \multicolumn{2}{|c|}{$R A S$ wild-type/BRAF wild-type } \\
\hline & $\begin{array}{l}\text { Panitumumab }+ \\
\text { mFOLFOX6 }(n=88)\end{array}$ & $\begin{array}{l}\text { Bevacizumab }+ \\
\operatorname{mFOLFOX6}(n=82)\end{array}$ & $\begin{array}{l}\text { Panitumumab }+ \\
\operatorname{mFOLFOX6}(n=77)\end{array}$ & $\begin{array}{l}\text { Bevacizumab }+ \\
\text { mFOLFOX6 }(n=79)\end{array}$ \\
\hline Male sex, $n(\%)$ & $58(66)$ & $56(68)$ & $50(65)$ & $55(70)$ \\
\hline Age, years - median (range) & $62(23-82)$ & $60(39-82)$ & $62(23-82)$ & $60(39-82)$ \\
\hline \multicolumn{5}{|l|}{ ECOG PS, $n(\%)$} \\
\hline 0 or 1 & $88(100)$ & $81(99)$ & $77(100)$ & $78(99)$ \\
\hline Missing & $0(0)$ & $1(1)$ & $0(0)$ & $1(1)$ \\
\hline \multicolumn{5}{|l|}{ Primary tumour diagnosis, $n(\%)$} \\
\hline Colon & $64(73)$ & $57(70)$ & $53(69)$ & $54(68)$ \\
\hline Rectum & $24(27)$ & $25(30)$ & $24(31)$ & $25(32)$ \\
\hline \multicolumn{5}{|l|}{ Primary tumour location, $n(\%)$} \\
\hline Left & $53(60)$ & $54(66)$ & $52(68)$ & $53(67)$ \\
\hline Right & $22(25)$ & $14(17)$ & $13(17)$ & $13(16)$ \\
\hline Unknown/unavailable & $13(15)$ & $14(17)$ & $12(16)$ & $13(16)$ \\
\hline \multicolumn{5}{|l|}{ Sites of metastases, $n(\%)$} \\
\hline Liver only & $23(26)$ & $22(27)$ & $21(27)$ & $22(28)$ \\
\hline Liver + other & $43(49)$ & $34(41)$ & $37(48)$ & $33(42)$ \\
\hline Other only & $22(25)$ & $26(32)$ & $19(25)$ & $23(29)$ \\
\hline Number of metastatic sites, median (range) & $2(1-5)$ & $2(0-4)$ & $2(1-5)$ & $2(0-4)$ \\
\hline $\begin{array}{l}\text { Sum of longest diameters of all target lesions, } \\
\mathrm{mm} \text { - mean (standard deviation) }{ }^{\mathrm{a}}\end{array}$ & $129.0(117.2)$ & $109.1(84.5)$ & $131.6(123.5)$ & $110.2(85.2)$ \\
\hline CEA levels, $\mu \mathrm{g} / \mathrm{L}$ - median (range) & $12.8(0-8543)$ & $15.2(1-4889)$ & $12.6(0-8453)$ & $15.6(1-4889)$ \\
\hline
\end{tabular}

${ }^{\text {a }}$ Sum of longest diameters of target lesions was missing/unknown for one patient in the bevacizumab group

CEA carcinoembryonic antigen, ECOG PS Eastern Cooperative Oncology Group performance status

were combined, achievement of $\geq 30$ versus $<30 \%$ ETS was associated with longer PFS (median $=12.9$ vs 9.8 months; $\mathrm{HR}=0.60(95 \% \mathrm{CI}=0.42-0.87) ; p=0.0065)$ and $\mathrm{OS}(\mathrm{me}-$ dian $=43.4$ vs 24.3 months; $\mathrm{HR}=0.44(95 \% \mathrm{CI}=0.30-0.65)$; $p<0.0001)$. Similar results were seen when combined data were analysed using the $\geq 20 \%$ ETS cutoff (median $\mathrm{PFS}=13.0$ vs 9.5 months; $\mathrm{HR}=0.55[95 \% \mathrm{CI}=0.37-$ $0.81] ; p=0.0029$; median $\mathrm{OS}=39.1$ vs 21.2 months; $\mathrm{HR}=0.39[95 \% \mathrm{CI}=0.26-0.59] ; p<0.0001)$.

\section{Safety}

In the $R A S$ WT safety analysis population, similar proportions of patients in the panitumumab and bevacizumab groups experienced AEs (100 vs 100\%), worst grade 3 or 4 AEs ( 90 vs $73 \%$ ), serious AEs (43 vs $40 \%$ ), fatal AEs (5 vs $9 \%$ ) and discontinuations (any drug) due to AEs (29 vs 30\%) (Online Resource Table A3). Overall, 20\% of patients discontinued panitumumab and $25 \%$ discontinued bevacizumab treatment due to an $\mathrm{AE}$. In the panitumumab arm, four fatal AEs (5\%) were reported: cardiac arrest, pneumonia aspiration, respiratory failure and sepsis $(n=1$ for each). In the bevacizumab arm, seven fatal AEs (9\%) were reported: cardiac arrest, sepsis, cardio-respiratory arrest, intestinal obstruction, intestinal perforation, septic shock and small intestinal perforation ( $n=1$ for each). Overall, 36 versus $3 \%$ of patients in the panitumumab versus bevacizumab groups had grade $\geq 3$ skin/subcutaneous tissue disorders; 5 versus $18 \%$ of patients in these groups, respectively, had grade $\geq 3$ vascular disorders. There was a $\geq 5 \%$ difference in incidence rate between treatment groups for the following grade $\geq 3$ AEs (panitumumab vs bevacizumab): rash (15 vs $0 \%$ ), hypomagnesaemia ( 8 vs $0 \%$ ), stomatitis ( 7 vs $0 \%$ ), decreased appetite (6 vs $1 \%$ ), dehydration (6 vs $1 \%$ ), acne (5 vs $0 \%$ ), dermatitis acneiform ( 5 vs $0 \%$ ), deep vein thrombosis ( 2 vs $8 \%$ ), and hypertension ( 0 vs $8 \%$ ) (Online Resource Table A3).

\section{Discussion}

The efficacy results from this final analysis of PEAK were consistent with the results of the primary analysis of this trial [9]. Patients with RAS WT tumours receiving panitumumab + mFOLFOX6 had longer PFS (12.8 vs 10.1 months, $p=0.029)$ and DoR (11.4 vs 9.0 months, $p=0.011$ ) compared with those receiving bevacizumab + mFOLFOX6. ORR (65 vs $60 \%$, $p=0.86)$ and resection rates (14 vs $11 \%$ ) were similar between treatments. Exploratory analyses of DpR (median 65 vs 
Table 2 Summary of progression-free survival, overall survival and objective response results

\begin{tabular}{|c|c|c|c|c|}
\hline & \multicolumn{2}{|l|}{$R A S$ wild-type } & \multicolumn{2}{|c|}{$R A S$ wild-type/ $B R A F$ wild-type } \\
\hline & $\begin{array}{l}\text { Panitumumab }+ \\
\text { mFOLFOX6 }(n=88)\end{array}$ & $\begin{array}{l}\text { Bevacizumab }+ \\
\operatorname{mFOLFOX6}(n=82)\end{array}$ & $\begin{array}{l}\text { Panitumumab }+ \\
\operatorname{mFOLFOX6}(n=77)\end{array}$ & $\begin{array}{l}\text { Bevacizumab }+ \\
\text { mFOLFOX6 }(n=79)\end{array}$ \\
\hline \multicolumn{5}{|l|}{ Progression-free survival } \\
\hline Patients with event, $n(\%)$ & $64(73)$ & $70(85)$ & $55(71)$ & $67(85)$ \\
\hline Median, months $(95 \% \mathrm{CI})$ & $12.8(10.7,15.1)$ & $10.1(9.0,12.7)$ & $13.1(11.6,16.2)$ & $10.1(9.0,12.7)$ \\
\hline $\operatorname{HR}(95 \% \mathrm{CI})$ & $0.68(0.48,0.96)$ & & $0.61(0.42,0.88)$ & \\
\hline$p$ value* & 0.029 & & 0.0075 & \\
\hline \multicolumn{5}{|l|}{ Overall survival } \\
\hline Patients with event, $n(\%)$ & $57(65)$ & $58(71)$ & $48(62)$ & $55(70)$ \\
\hline Median, months $(95 \% \mathrm{CI})$ & $36.9(27.9,46.1)$ & $28.9(23.3,32.0)$ & $41.3(31.6,46.7)$ & $28.9(23.9,33.1)$ \\
\hline $\mathrm{HR}(95 \% \mathrm{CI})$ & $0.76(0.53,1.11)$ & & $0.70(0.48,1.04)$ & \\
\hline$p$ value* & 0.15 & & 0.08 & \\
\hline \multicolumn{5}{|l|}{ Subsequent therapy } \\
\hline $\begin{array}{l}\text { Anti-EGFR mAb, } \\
n(\%)\end{array}$ & $28(32)$ & $41(50)$ & $27(35)$ & $40(51)$ \\
\hline $\begin{array}{l}\text { Anti-VEGF mAb, } \\
n(\%)\end{array}$ & $44(50)$ & $31(38)$ & $39(51)$ & $30(38)$ \\
\hline \multicolumn{5}{|l|}{ Objective response $^{\dagger}$} \\
\hline Responders, ${ }^{\ddagger} n$ & 57 & 49 & 50 & 48 \\
\hline ORR, ${ }^{\ddagger} \%(95 \% \mathrm{CI})$ & $65(54,75)$ & $60(49,71)$ & $65(53,75)$ & $62(50,72)$ \\
\hline $\begin{array}{l}\text { Difference in rates, \% } \\
(95 \% \mathrm{CI})\end{array}$ & $4.3(-10.9,19.3)$ & & $3.4(-12.5,19.0)$ & \\
\hline Odds ratio $^{\mathbb{I}}(95 \% \mathrm{CI})$ & $1.12(0.56,2.22)$ & & $1.11(0.54,2.27)$ & \\
\hline$p$ value $^{\S}$ & 0.86 & & 0.90 & \\
\hline
\end{tabular}

$C I$ confidence interval, $E G F R$ epidermal growth factor receptor, $H R$ hazard ratio, $m A b$ monoclonal antibody, ORR objective response rate, RECIST Response Evaluation Criteria In Solid Tumours, VEGF vascular endothelial growth factor, WT wild type

*From stratified Cox model

${ }^{\dagger}$ For the objective response analysis, $n=81$ for the RAS WT bevacizumab group and $n=78$ for the RAS WT/BRAF WT bevacizumab group

\# As assessed by RECIST

${ }^{\mathbb{I}}$ Defined as the odds of having an objective response in the panitumumab + mFOLFOX6 arm relative to the odds in the bevacizumab + mFOLFOX6 arm

$\S$ From stratified exact test

$46 \%, p=0.0018)$ and $\operatorname{ETS}(\geq 30 \%$ ETS 64 vs $45 \%, p=0.052)$ suggested improvements in these measures for the panitumumab versus bevacizumab group. In line with previous reports $[10,11,14,15]$, ETS appeared to be associated with improved PFS and OS, irrespective of first-line treatment received. A limitation to this study is that radiologic assessment was performed by the investigator and no independent radiologic review was performed.

In the RAS WT population, median OS was 8 months longer in the panitumumab group (36.9 vs 28.9 months; $\mathrm{HR}=0.76[95 \% \mathrm{CI}=0.53-1.11] ; p=0.15)$. Median OS was further extended in patients with $R A S$ WT/BRAF WT tumours receiving panitumumab (41.3 months), but remained the same as for the RAS WT population in RAS WT/BRAFWT patients receiving bevacizumab (28.9 months). This is of note because patients with $B R A F$ MT mCRC have a poor prognosis [12] and there is a small imbalance in the number of patients with BRAF MT tumours in this study (panitumumab arm $n=11$; bevacizumab arm $n=3$ ). However, the primary endpoint in PEAK was not OS and this trial was not powered to detect an OS increase, which may explain why the observed difference between arms was not statistically significant. The overall findings from PEAK nonetheless suggest an OS benefit for EGFRIs + chemotherapy versus bevacizumab + chemotherapy, which is consistent with results from FIRE-3 [16] and a congress presentation of limited data for the FOLFOX arm of the CALGB/SWOG 80405 study (full publication not yet available) [17]. This is also in line with results from two recent meta-analyses of these trials, which included primary analysis data from PEAK. Both meta-analyses demonstrated significantly improved ORR and OS with first-line 
Fig. 2 Kaplan-Meier estimates of a Progression-free survival and $\mathbf{b}$ Overall survival (RAS wild-type population)

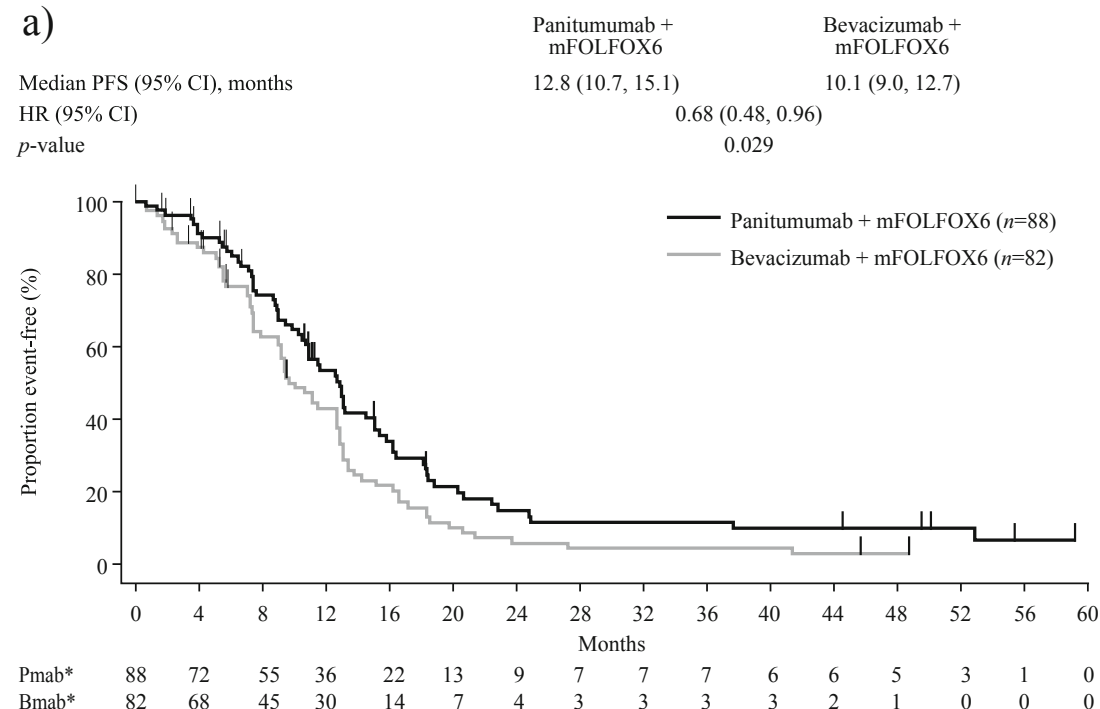

b)
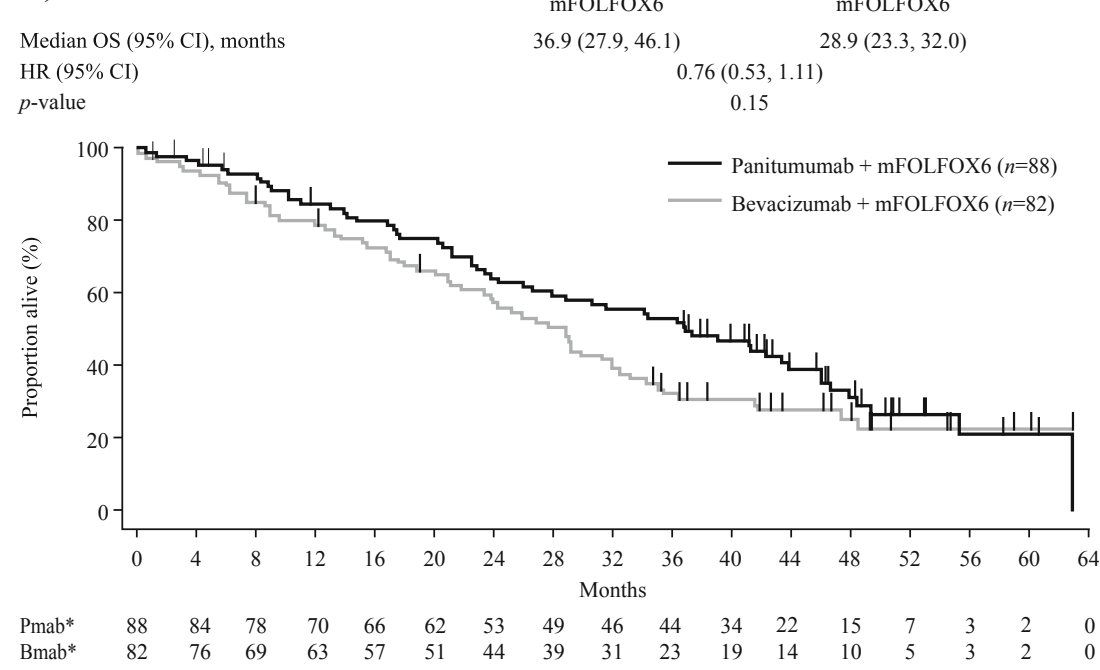

Censor indicated by vertical bar. $*+$ mFOLFOX6

Bmab, bevacizumab; CI, confidence intervals; HR, hazard ratio; OS, overall survival; PFS, progression-free survival; Pmab, panitumumab
anti-EGFR compared with anti-VEGF therapy in patients with RAS WT mCRC $[18,19]$. The HR $(95 \% \mathrm{CI})$ for OS was 0.77 $(0.63-0.95)$ in favour of the EGFRI in both analyses. Similar results were also observed in a meta-analysis including seven first-line trials comparing panitumumab or cetuximab plus chemotherapy with chemotherapy alone or chemotherapy plus bevacizumab [20].

The median OS of 41.3 months achieved in patients with RAS WT/BRAF WT $\mathrm{mCRC}$ receiving panitumumab in PEAK, compares well with other data for first-line biologics combined with doublet chemotherapy [12, 13]. Data have recently been reported for triplet chemotherapy combined with biologics in several studies including TRIBE [13, 21] (bevacizumab), TRIP [22] and VOLFI [23] (panitumumab) and MACBETH [24] (cetuximab), indicating that
FOLFOXIRI may offer superior efficacy over doublet chemotherapy in the first-line setting. However, improved outcomes are achieved at the expense of increased grade 3/4 toxicity, including neutropenia, diarrhoea, stomatitis and neurotoxicity, which may limit the use of such regimens in clinical practice.

Although the OS results from PEAK are now more mature (68 and 66\% deaths in the RAS WT and RAS WT/BRAF WT populations, respectively), these results need to be considered in context of the fact that PEAK is a phase II trial. There are several possible reasons for the prolonged OS observed. For example, the improved DoR in the panitumumab + mFOLFOX6 arm could be linked with the improved PFS and numerically improved OS seen in this arm. Likewise, the improved DpR, ETS and tumour load changes in the panitumumab versus bevacizumab arm could also play a role 
Table 3 Summary of response- and resection-related efficacy results ( $R A S$ wild-type population)

\begin{tabular}{|c|c|c|}
\hline & Panitumumab + mFOLFOX6 $(n=88)$ & Bevacizumab + mFOLFOX6 $(n=82)^{*}$ \\
\hline Median DoR, months $(95 \% \mathrm{CI})$ & $11.4(10.0,16.3)$ & $9.0(7.6,9.5)$ \\
\hline $\mathrm{HR}^{\dagger}(95 \% \mathrm{CI})$ & $0.59(0.39,0.88)$ & \\
\hline$p$ value & 0.011 & \\
\hline Median TTR, months $(95 \% \mathrm{CI})$ & $2.3(1.9,3.7)$ & $3.8(2.1,5.7)$ \\
\hline $\mathrm{HR}^{\dagger}(95 \% \mathrm{CI})$ & $1.19(0.81,1.74)$ & \\
\hline$p$ value & 0.37 & \\
\hline Median DpR, \% (Q1, Q3) & $65.0(45.7,89.5)$ & $46.3(29.5,63.3)$ \\
\hline$p$ value $\mathrm{e}^{\mathrm{Tl}}$ & 0.0018 & \\
\hline Any resection, $n(\%)$ & $12(14)$ & $9(11)$ \\
\hline Liver only & $9(75)$ & $6(67)$ \\
\hline Complete resection & $9(10)$ & $7(9)$ \\
\hline Liver only & $7(78)$ & $6(86)$ \\
\hline Time to resection, months - median (range) & $5.1(3-19)$ & $4.4(3-12)$ \\
\hline \multicolumn{3}{|l|}{ Progression-free at 6 months, $n(\%)$} \\
\hline Patients with resection & $8 / 12(67)$ & $4 / 9(44)$ \\
\hline Patients without resection & $45 / 76(59)$ & $48 / 73(66)$ \\
\hline \multicolumn{3}{|l|}{ Alive at 2 years, $n(\%)$} \\
\hline Patients with resection & $8 / 12(67)$ & 8/9 (89) \\
\hline Patients without resection & $18 / 76(24)$ & $12 / 73(16)$ \\
\hline
\end{tabular}

$C I$ confidence interval, $D o R$ duration of response, $D p R$ depth of response, $H R$ hazard ratio, TTR time to response

*For DoR, TTR and DpR analyses, $n=81$ for the bevacizumab group

${ }^{\dagger}$ HRs are presented as panitumumab + mFOLFOX6 : bevacizumab + mFOLFOX6. A value $<1.0$ indicates a lower average event rate and longer time to event for panitumumab + mFOLFOX6 relative to bevacizumab + mFOLFOX6

* For treatment effect

II $p$ value from Wilcoxon test

$\S$ Percentage is calculated using the number of patients who had resection as the denominator

${ }^{\wedge}$ Percentage is calculated using the number of patients who had complete resection as the denominator

in improving long-term outcomes, as has been suggested in other reports $[11,25]$. It is unlikely that the numeric, betweentreatment OS differences were driven by resection, as resection rates in patients with $R A S$ WT tumours were balanced between groups. It is also unlikely to be driven by differences in tumour side as the proportion of patients with left- and right-sided disease was well-balanced between treatment groups. As was suggested in FIRE-3 [26], the impact of treatment sequence could also be important, given that the proportions of patients switching biologic approach were balanced
Fig. 3 Mean $(95 \%$ confidence interval) percentage change from baseline in tumour load (sum of all target lesions) over time ( $R A S$ wild-type population)

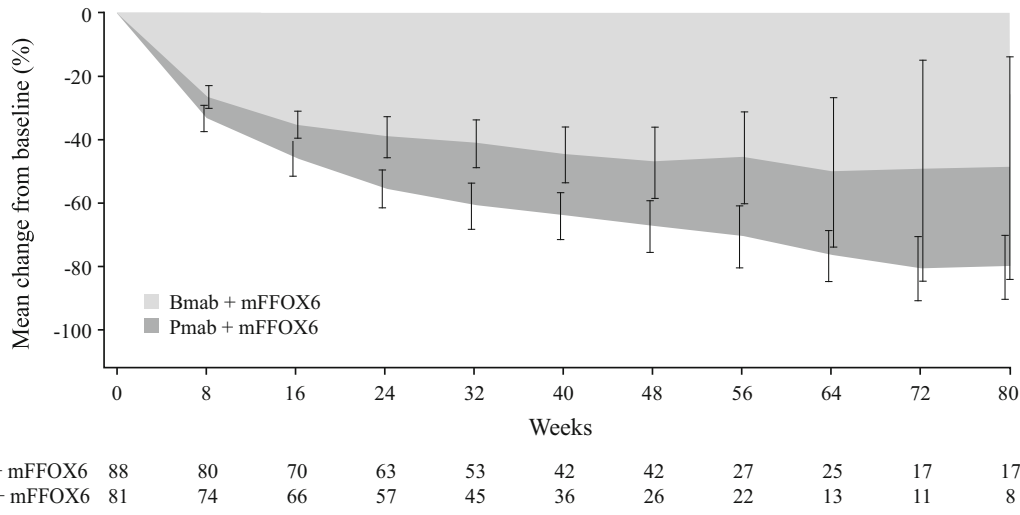

* Sum of all target lesions - includes those patients evaluable for objective response who had baseline tumour shrinkage data Bmab, bevacizumab; Pmab, panitumumab; mFFOX6, mFOLFOX6 
Table 4 Progression-free and overall survival outcomes by tumour shrinkage at week 8 ( $R A S$ wild-type population - between treatment comparisons)

\begin{tabular}{|c|c|c|c|c|}
\hline & \multicolumn{4}{|c|}{ Tumour shrinkage at week 8} \\
\hline & $\begin{array}{l}\text { Panitumumab + } \\
\text { mFOLFOX6 }\end{array}$ & $\begin{array}{l}\text { Bevacizumab + } \\
\text { mFOLFOX6 }\end{array}$ & $\begin{array}{l}\text { Panitumumab + } \\
\text { mFOLFOX6 }\end{array}$ & $\begin{array}{l}\text { Bevacizumab + } \\
\text { mFOLFOX6 }\end{array}$ \\
\hline & \multicolumn{2}{|l|}{$<30 \%$} & \multicolumn{2}{|l|}{$\geq 30 \%$} \\
\hline $\begin{array}{l}\text { Patients with shrinkage, } \\
n(\%)\end{array}$ & $29(36)$ & $41(55)$ & $51(64)$ & $33(45)$ \\
\hline Odds ratio* $(95 \% \mathrm{CI})$ & \multicolumn{4}{|l|}{$1.99(0.99,4.10)$} \\
\hline$p$ value & \multicolumn{4}{|l|}{0.052} \\
\hline $\begin{array}{l}\text { Median PFS, months } \\
(95 \% \mathrm{CI})\end{array}$ & $11.6(7.5,15.4)$ & $9.7(7.5,12.9)$ & $13.0(10.9,18.1)$ & $11.1(9.0,16.6)$ \\
\hline $\mathrm{HR}(95 \% \mathrm{CI})$ & \multicolumn{2}{|l|}{$0.79(0.45,1.38)$} & \multicolumn{2}{|l|}{$0.74(0.45,1.23)$} \\
\hline$p$ value & \multicolumn{2}{|l|}{0.40} & \multicolumn{2}{|l|}{0.24} \\
\hline $\begin{array}{l}\text { Median OS, months } \\
\quad(95 \% \mathrm{CI})\end{array}$ & $34.2(17.5,42.3)$ & $23.9(20.1,29.0)$ & $43.8(36.4,63.0)$ & $35.1(29.9, \mathrm{NE})$ \\
\hline $\mathrm{HR}(95 \% \mathrm{CI})$ & \multicolumn{2}{|l|}{$0.75(0.43,1.31)$} & \multicolumn{2}{|l|}{$0.77(0.42,1.42)$} \\
\hline$p$ value & \multicolumn{2}{|l|}{0.31} & \multicolumn{2}{|l|}{0.41} \\
\hline & \multicolumn{2}{|l|}{$<20 \%$} & \multicolumn{2}{|l|}{$\geq 20 \%$} \\
\hline $\begin{array}{l}\text { Patients with shrinkage, } \\
n(\%)\end{array}$ & $20(25)$ & $28(38)$ & $60(75)$ & $46(62)$ \\
\hline Odds ratio* $(95 \% \mathrm{CI})$ & \multicolumn{4}{|l|}{$1.67(0.78,3.58)$} \\
\hline$p$ value & \multicolumn{4}{|l|}{0.21} \\
\hline $\begin{array}{l}\text { Median PFS, months } \\
\quad(95 \% \mathrm{CI})\end{array}$ & $9.8(4.2,15.4)$ & $9.5(7.4,12.7)$ & $13.1(10.9,16.2)$ & $11.3(9.2,13.6)$ \\
\hline $\mathrm{HR}(95 \% \mathrm{CI})$ & $0.99(0.50,1.95)$ & & $0.70(0.45,1.08)$ & \\
\hline$p$ value & 0.97 & & 0.11 & \\
\hline $\begin{array}{l}\text { Median OS, months } \\
\quad(95 \% \mathrm{CI})\end{array}$ & $21.2(14.1,41.2)$ & $21.8(15.3,28.9)$ & $43.4(36.4,55.4)$ & $32.5(27.7,47.4)$ \\
\hline $\operatorname{HR}(95 \% \mathrm{CI})$ & $0.81(0.42,1.57)$ & & $0.73(0.44,1.19)$ & \\
\hline$p$ value & 0.53 & & 0.21 & \\
\hline
\end{tabular}

*Odds ratio is defined as the odds of having $\geq 30$ or $\geq 20 \%$ tumour shrinkage in the panitumumab + mFOLFOX6 arm relative to the odds in the bevacizumab + mFOLFOX6 arm

$C I$ confidence intervals, $H R$ hazard ratio, $N E$ not evaluable, $O S$ overall survival, $P F S$ progression-free survival

between arms (i.e. EGFRI to VEGFI or VEGFI to EGFRI). Preclinical data suggest that first-line EGFRI treatment may sensitise tumours to subsequent therapy, whereas first-line VEGFI may desensitise tumours [27-29]. This provides a biological rationale for first-line EGFRI therapy followed by second-line VEGFI therapy.

As well as comparative efficacy, safety/tolerability and costeffectiveness of the available treatments need to be considered when choosing first-line therapy for patients with $R A S$ WT mCRC. Updated data for the RAS WT population in PEAK suggest that the safety profiles of the two treatments are consistent with previously reported studies and no new toxicities or safety signals were identified. The main tolerability differences were due to known class effects of these treatments, e.g. skin rash/toxicities and hypomagnesaemia with the EGFRI and deep vein thrombosis and hypertension with bevacizumab. Discontinuation rates due to AEs were similar between arms (29 vs 30\%, respectively).
Tumour biomarker status is increasingly being used to guide treatment decisions in mCRC and is a rapidly developing area of research. By targeting drugs only to those patients most likely to benefit, predictive biomarkers may improve clinical outcomes and also potentially reduce overall treatment costs. The availability of banked tumour samples from clinical studies is important to permit prospective, prospective-retrospective or retrospective analyses to be performed to test new biomarker hypotheses [30]. Analysis of data from the phase III PRIME study of panitumumab + FOLFOX4 confirmed the importance of RAS testing beyond $K R A S$ in patients with $\mathrm{mCRC}$ and was possible due to the availability of $R A S$ data for $90 \%$ of the KRAS WT tumour samples [12]. The RAS ascertainment rate in the PEAK study was also high $(82 \%)$ in comparison to currently available data from other first-line mCRC studies comparing EGFRIs versus VEGFIs (FIRE-3 $=69 \%$ [16]; CALGB $80405=55 \%$ [17]), highlighting the importance of including the availability of 
paraffin-embedded tumour tissue for biomarker testing as a requirement for study entry. Mandating baseline biopsy and planning the conduct of prospective/prespecified biomarker analyses in clinical trials, can improve the robustness of the study data and future analyses. While tumour $R A S$ status is undoubtedly a valuable predictive marker for response to EGFRIs, it remains important to investigate the roles of other potential biomarkers that could improve patient selection (e.g. epiregulin/amphiregulin, PIK3CA, PTEN). Data from the PRIME study indicated that $B R A F$ mutations are linked with poor outcomes irrespective of treatment received [12]. RAS mutations do not appear to be prognostic for patients with mCRC.

In summary, ORR by RECIST is the accepted standard for assessing response in solid tumours, but has limitations in terms of assessing dynamic tumour burden changes. Although ORRs were similar between treatments in PEAK, tumour responses in the panitumumab arm appeared to occur earlier, last longer and be deeper compared with those occurring in the bevacizumab arm in these exploratory analyses. More patients receiving panitumumab + mFOLFOX6 versus bevacizumab + mFOLFOX6 had $\geq 30 \%$ ETS; ETS of $\geq 30$ or $\geq 20 \%$ appeared to be associated with improved PFS and OS, irrespective of treatment received. PFS was also significantly improved for patients with RAS WT mCRC receiving panitumumab + mFOLFOX6 versus bevacizumab + mFOLFOX6. For panitumumab versus bevacizumab, median OS was 8.0 months longer in the RAS WT (36.9 vs 28.9 months) and 12.4 months longer in the RAS WT/BRAF WT (41.3 vs 28.9 months) populations, respectively (neither difference being statistically significant). Based on these data, panitumumab + mFOLFOX6 can be considered an effective first-line treatment for patients with RAS WT mCRC. A phase III trial is warranted to more fully evaluate any potential differences between panitumumab and bevacizumab treatment in the first-line setting.

Acknowledgements The authors wish to acknowledge all of the patients who participated in the PEAK (20070509) study, the PEAK study investigators and their staff for their participation in the conduct and reporting of this study, the study and biostatistics team at Amgen, and the medical writing support provided by Dawn Batty $\mathrm{PhD}$ of Bioscript Medical Ltd. (funded by Amgen [Europe] GmbH). Amgen Inc. sponsored the PEAK study and was involved in design, conduct, data collection, analysis and data interpretation.

Funding Amgen Inc.

\section{Compliance with ethical standards}

Conflict of interest FR has acted on advisory boards and received research funding from Amgen, during the course of this study. He has also acted on advisory boards or received research funding from Bayer, Lilly, Merck-Serono, Merck Sharp and Dohme, Roche, Sanofi, and Servier, outside of the submitted work. MK reports personal fees from Amgen and Roche, outside the submitted work. JRH reports personal fees from Amgen and Roche, outside the submitted work. IS has acted on advisory boards/received honoraria from Amgen, Roche, MerckSerono, Sanofi, Bayer, Ipsen and Novartis. FF has acted on advisory boards/received honoraria from Novartis and Nutricia, and received research grants from Amgen, outside the submitted work. GF reports grants and personal fees from Amgen, during the course of the study. JLC reports research grants from Amgen, outside the submitted work. XG is an employee of Amgen Inc. GD is an employee of Amgen (Europe) $\mathrm{GmbH}$. LSS has acted as a consultant and on a speaker's bureau for Amgen, outside the submitted work.

Statement of human rights PEAK (NCT00819780) was conducted in compliance with the Declaration of Helsinki. The study protocol was approved by an independent ethics committee at each participating study centre.

Informed consent All patients provided informed consent before any study procedures were performed.

Open Access This article is distributed under the terms of the Creative Commons Attribution 4.0 International License (http:// creativecommons.org/licenses/by/4.0/), which permits unrestricted use, distribution, and reproduction in any medium, provided you give appropriate credit to the original author(s) and the source, provide a link to the Creative Commons license, and indicate if changes were made.

\section{References}

1. National Comprehensive Cancer Network ${ }^{\circledR}$ (2015) Clinical practice guidelines in oncology $\left(\mathrm{NCCN}\right.$ Guidelines $\left.{ }^{\circledR}\right)$ : colon cancer version 1.2015. Available at: www.nccn.org/. Access date 15 March 2017

2. Van Cutsem E, Cervantes A, Nordlinger B, Arnold D (2014) Metastatic colorectal cancer: ESMO clinical practice guidelines for diagnosis, treatment and follow-up. Ann Oncol 25(Suppl 3): iii1-iii9

3. Koike J, Ushigome M, Funahashi K, Shiokawa H, Kaneko T, Arai K, Matsuda S, Kagami S, Suzuki T, Kurihara A, Shimada H, Kaneko H (2014) Significance of KRAS mutation in patients receiving mFOLFOX6 with or without bevacizumab for metastatic colorectal cancer. Hepato-Gastroenterology 61:2222-2226

4. Price TJ, Bruhn MA, Lee CK, Hardingham JE, Townsend AR, Mann KP, Simes J, Weickhardt A, Wrin JW, Wilson K, Gebski V, Van HG, Robinson B, Cunningham D, Tebbutt NC (2015) Correlation of extended RAS and PIK3CA gene mutation status with outcomes from the phase III AGITG MAX STUDY involving capecitabine alone or in combination with bevacizumab plus or minus mitomycin $\mathrm{C}$ in advanced colorectal cancer. Br J Cancer 112:963-970

5. European Medicines Agency (2015) Vectibix ${ }^{\circledR}$ (panitumumab) Summary of Product Characteristics. Available at: http://www. ema.europa.eu/docs/en_GB/document_library/EPAR__Product Information/human/000741/WC500047710.pdf. Access date $1 \overline{5}$ March 2017

6. Amgen Inc. (2015) Vectibix® prescribing information. Available at: http://pi.amgen.com/united_states/vectibix/vectibix_pi.pdf. Access date 15 March 2017

7. European Medicines Agency (2015) Avastin ${ }^{\circledR}$ (bevacizumab) summary of product characteristics. Available at: http://www.ema. europa.eu/docs/en_GB/document_library/EPAR__Product Information/human/000582/WC500029271.pdf. Access date $1 \overline{5}$ March 2017

8. Genentech Inc. (2015) Avastin ${ }^{\circledR}$ (bevacizumab) prescribing information; South San Francisco, CA: Genentech, Inc. Available at: 
http://www.gene.com/gene/products/information/pdf/avastinprescribing.pdf. Access date 15 March 2017

9. Schwartzberg LS, Rivera F, Karthaus M, Fasola G, Canon JL, Hecht JR, Yu H, Oliner KS, Go WY (2014) PEAK: a randomized, multicenter phase II study of panitumumab plus modified fluorouracil, leucovorin, and oxaliplatin (mFOLFOX6) or bevacizumab plus mFOLFOX6 in patients with previously untreated, unresectable, wild-type KRAS exon 2 metastatic colorectal cancer. J Clin Oncol 32:2240-2247

10. Heinemann V, Modest DP, Fischer von Weikersthal L, Decker T, Kiani A, Vehling-Kaiser U, Al-Batran S, Heintges T, Lerchenmüller C, Kahl C, Seipelt G, Kullmann F, Stauch M, Scheithauer W, Held S, Giessen C, Möhler M, Stintzing S (2014) Independent radiological evaluation of objective response, early tumour shrinkage, and depth of response in FIRE-3 (AIO KRK-0306). Ann Oncol 25(Suppl 4):ii117 (Abstract O-0030)

11. Cremolini C, Loupakis F, Antoniotti C, Lonardi S, Masi G, Salvatore L, Cortesi E, Tomasello G, Spadi R, Zaniboni A, Tonini G, Barone C, Vitello S, Longarini R, Bonetti A, D'Amico M, Di DS, Granetto C, Boni L, Falcone A (2015) Early tumor shrinkage and depth of response predict long-term outcome in metastatic colorectal cancer patients treated with first-line chemotherapy plus bevacizumab: results from phase III TRIBE trial by the Gruppo Oncologico del Nord Ovest. Ann Oncol 26:1188-1194

12. Douillard JY, Oliner KS, Siena S, Tabernero J, Burkes R, Barugel M, Humblet Y, Bodoky G, Cunningham D, Jassem J, Rivera F, Kocákova I, Ruff P, Blasinska-Morawiec M, Smakal M, Canon JL, Rother M, Williams R, Rong A, Wiezorek J, Sidhu R, Patterson SD (2013) PanitumumabFOLFOX4 treatment and RAS mutations in colorectal cancer. N Engl J Med 369:1023-1034

13. Cremolini C, Loupakis F, Antoniotti C, Lupi C, Sensi E, Lonardi S, Mezi S, Tomasello G, Ronzoni M, Zaniboni A, Tonini G, Carlomagno C, Allegrini G, Chiara S, D'Amico M, Granetto C, Cazzaniga M, Boni L, Fontanini G, Falcone A (2015) FOLFOXIRI plus bevacizumab versus FOLFIRI plus bevacizumab as first-line treatment of patients with metastatic colorectal cancer: updated overall survival and molecular subgroup analyses of the open-label, phase 3 TRIBE study. Lancet Oncol 16:1306-1315

14. Piessevaux H, Buyse M, Schlichting M, Van CE, Bokemeyer C, Heeger S, Tejpar S (2013) Use of early tumor shrinkage to predict long-term outcome in metastatic colorectal cancer treated with cetuximab. J Clin Oncol 31:3764-3775

15. Douillard JY, Siena S, Peeters M, Koukakis R, Terwey JH, Tabernero J (2015) Impact of early tumour shrinkage and resection on outcomes in patients with wild-type RAS metastatic colorectal cancer. Eur J Cancer 51:1231-1242

16. Heinemann V, von Weikersthal LF, Decker T, Kiani A, VehlingKaiser U, Al-Batran SE, Heintges T, Lerchenmuller C, Kahl C, Seipelt G, Kullmann F, Stauch M, Scheithauer W, Hielscher J, Scholz M, Muller S, Link H, Niederle N, Rost A, Hoffkes HG, Moehler M, Lindig RU, Modest DP, Rossius L, Kirchner T, Jung A, Stintzing S (2014) FOLFIRI plus cetuximab versus FOLFIRI plus bevacizumab as first-line treatment for patients with metastatic colorectal cancer (FIRE-3): a randomised, open-label, phase 3 trial. Lancet Oncol 15:1065-1075

17. Lenz H, Niedzwiecki D, Innocenti F, Blanke C, Mahoney MR, O'Neil BH, Shaw JE, Polite B, Hochster H, Atkins J, Goldberg R, Mayer R, Schilsky R, Bertagnolli M, Venook A (2014) CALGB/SWOG 80405: phase III trial of irinotecan/5-FU/leucovorin (FOLFIRI) or oxaliplatin/ 5-FU/leucovorin (mFOLFOX6) with bevacizumab (BV) or cetuximab (CET) for patients (pts) with expanded ras analyses untreated metastatic adenocarcinoma of the colon or rectum (mCRC). Ann Oncol 25(Suppl 4):ii117 (Abstract 501O)
18. Khattak MA, Martin H, Davidson A, Phillips M (2015) Role of first-line anti-epidermal growth factor receptor therapy compared with anti-vascular endothelial growth factor therapy in advanced colorectal cancer: a meta-analysis of randomized clinical trials. Clin Colorectal Cancer 14:81-90

19. Heinemann V, Rivera F, O'Neil BH, Stintzing S, Koukakis R, Terwey JH, Douillard JY (2016) A study level metaanalysis of efficacy data from head-to-head first-line trials of epidermal growth factor receptor inhibitors vs bevacizumab in patients with RAS wild-type metastatic colorectal cancer. Eur J Cancer 67:11-20

20. Pietrantonio F, Cremolini C, Petrelli F, Di Bartolomeo M, Loupakis F, Maggi C, Antoniotti C, de Braud F, Falcone A, Iacovelli R (2015) First-line anti-EGFR monoclonal antibodies in panRAS wild-type metastatic colorectal cancer: a systematic review and meta-analysis. Crit Rev Oncol Hematol 96:156-166

21. Loupakis F, Cremolini C, Masi G, Lonardi S, Zagonel V, Salvatore L, Cortesi E, Tomasello G, Ronzoni M, Spadi R, Zaniboni A, Tonini G, Buonadonna A, Amoroso D, Chiara S, Carlomagno C, Boni C, Allegrini G, Boni L, Falcone A (2014) Initial therapy with FOLFOXIRI and bevacizumab for metastatic colorectal cancer. $\mathrm{N}$ Engl J Med 371:1609-1618

22. Fornaro L, Lonardi S, Masi G, Loupakis F, Bergamo F, Salvatore L, Cremolini C, Schirripa M, Vivaldi C, Aprile G, Zaniboni A, Bracarda S, Fontanini G, Sensi E, Lupi C, Morvillo M, Zagonel V, Falcone A (2013) FOLFOXIRI in combination with panitumumab as first-line treatment in quadruple wild-type (KRAS, NRAS, HRAS, BRAF) metastatic colorectal cancer patients: a phase II trial by the Gruppo Oncologico Nord Ovest (GONO). Ann Oncol 24:2062-2067

23. Martens U, Wessendorf S, Riera Knorrenschild J, Buechner-Steudel P, Florschuetz A, Atzpodien J, Keller R, Greeve J, Kanzler S, Ettrich T, Lindig U, Egger M, Hebart H, Geissler M (2015) AIOKRK-0109: a randomized phase II trial of panitumumab plus FOLFOXIRI or FOLFOXIRI alone as 1st-line treatment in RASwild-type metastatic colorectal cancer (mCRC). Ann Oncol 6(Suppl 4) Abstract 2049

24. Cremolini C, Loupakis F, Salvatore L, Lonardi S, Battaglin F, Gamucci T, Grande R, Di Fabio F, Rojas Llimpe FL, Ferrari L, Casagrande M, Lucchesi S, Tonini G, Banzi M, Valsuani C, Corsi DC, Fontanini G, Tomcikova D, Falcone A (2014) Modified FOLFOXIRI plus cetuximab (cet) as induction treatment in unresectable metastatic colorectal cancer (mCRC) patients (pts): preliminary results of the phase II randomized Macbeth trial by GONO group. J Clin Oncol 32(Suppl 5S) Abstract 3596

25. Heinemann V, Stintzing S, Modest DP, Giessen-Jung C, Michl M, Mansmann UR (2015) Early tumour shrinkage (ETS) and depth of response (DpR) in the treatment of patients with metastatic colorectal cancer (mCRC). Eur J Cancer 51:1927-1936

26. Modest DP, Stintzing S, von Weikersthal LF, Decker T, Kiani A, Vehling-Kaiser U, Al-Batran SE, Heintges T, Lerchenmuller C, Kahl C, Seipelt G, Kullmann F, Stauch M, Scheithauer W, Held S, Mohler M, Jung A, Kirchner T, Heinemann V (2015) Impact of subsequent therapies on outcome of the FIRE-3/AIO KRK0306 trial: first-line therapy with FOLFIRI plus cetuximab or bevacizumab in patients with KRAS wild-type tumors in metastatic colorectal cancer. J Clin Oncol 33:3718-3726

27. Viloria-Petit A, Crombet $\mathrm{T}$, Jothy $\mathrm{S}$, Hicklin $\mathrm{D}$, Bohlen $\mathrm{P}$, Schlaeppi JM, Rak J, Kerbel RS (2001) Acquired resistance to the antitumor effect of epidermal growth factor receptorblocking antibodies in vivo: a role for altered tumor angiogenesis. Cancer Res 61:5090-5101

28. Ciardiello F, Bianco R, Caputo R, Caputo R, Damiano V, Troiani T, Melisi D, De Vita F, De Placido S, Bianco AR, Tortora G (2004) Antitumor activity of ZD6474, a vascular endothelial growth factor 
receptor tyrosine kinase inhibitor, in human cancer cells with acquired resistance to antiepidermal growth factor receptor therapy. Clin Cancer Res 10:784-793

29. Wainberg ZA, Drakaki A (2015) The importance of optimal drug sequencing in metastatic colorectal cancer: biological rationales for the observed survival benefit conferred by first-line treatment with EGFR inhibitors. Expert Opin Biol Ther 15:1205-1220

30. Peeters M, Karthaus M, Rivera F, Terwey JH, Douillard JY (2015)

Panitumumab in metastatic colorectal cancer: the importance of tumour RAS status. Drugs 75:731-748 